\title{
Noise delayed decay of unstable states: theory versus numerical simulations
}

\author{
N V Agudov $\uparrow+$, R Mannella§, A V Safonov $\dagger+$ and B Spagnolo $\dagger \|$ \\ $\dagger$ INFM - Unità di Palermo, Group of Interdisciplinary Physics and Dipartimento di \\ Fisica e Tecnologie Relative, Università di Palermo, Viale delle Scienze, I-90128 \\ Palermo, Italia \\ $\ddagger$ Radiophysical Department, State University of N.Novgorod, 23 Gagarin Ave., \\ Nizhny Novgorod, 603600, Russia \\ $\S$ Dipartimento di Fisica and INFM UdR Pisa, Università degli Studi di Pisa, 56100 \\ Pisa, Italia
}

\begin{abstract}
We study the noise delayed decay of unstable nonequilibrium states in nonlinear dynamical systems within the framework of the overdamped Brownian motion model. We give the exact expressions for the decay times of unstable states for polynomial potential profiles and obtain nonmonotonic behavior of the decay times as a function of the noise intensity for the unstable nonequilibrium states. The analytical results are compared with numerical simulations.
\end{abstract}

PACS numbers: 05.40.-a,02.50.-r,05.10.Gg 


\section{Introduction}

In the last two decades a large variety of noise induced phenomena have been discovered in far from equilibrium nonlinear systems. Among them there are stochastic resonance [1, resonant activation [2], noise enhanced stability [3]-[5] and noise delayed decay [6]- [6]. Recently it was predicted theoretically that the presence of additive noise may increase the average escape time from metastable states [4, 5, 9, 10, and from unstable states [7, 8. Moreover the noise can enhance the stability of an unstable fixed point in nonlinear discrete maps [6, 11]. These resonance-like phenomena show a nonmonotonic behaviour of the average escape time as a function of the noise intensity. This means that by varying the noise intensity we can lengthen or shorten the lifetime of unstable states. In previous studies in fact was found that fluctuations can only accelerate the escape time from unstable states [12-[15]. In the present work we study the phenomenon of Noise Delayed Decay (NDD) in more detail by analytical and numerical methods. Besides the Mean First Passage Time (MFPT), which quantifies only one of many possible characteristics of the decay process from unstable states, we consider the Nonlinear Relaxation Time (NLRT). The NLRT takes into account the inverse probability current directed from the stable state to the unstable one, which is neglected by the MFPT. The analytical calculations show that NDD effect for the NLRT is much greater than that for MFPT [8]. In the present paper these analytical predictions are verified with numerical simulations. We show that the NLRT can be increased by the noise, when the noise intensity is varied in a very wide range. We consider the model of one-dimensional overdamped Brownian motion in the potential field of force

$$
\frac{d x}{d t}=-\frac{d \Phi(x)}{\eta d x}+\xi(t)
$$

Here $x$ is the coordinate of the Brownian particle or the representative phase point denoting the state of the system, $\Phi(x)$ is the potential describing the system itself, $\xi(t)$ is the white Gaussian noise, with $\langle\xi(t)\rangle=0,\langle\xi(t) \xi(t+\tau)\rangle=2 q \delta(\tau) / \eta, 2 q / \eta$ is the intensity of fluctuations, $\eta$ is the coefficient of equivalent viscosity, and $q=k T$

is the energy temperature of fluctuations. The simplest case where the NDD appears is the parabolic potential. This potential profile was considered in theoretical papers concerning the decay from equilibrium unstable states [12, 13], [15]-[18]

$$
\Phi(x)=-a x^{2} / 2 .
$$

The initial state is an unstable equilibrium state, if $x_{0}=0$, and it is an unstable nonequilibrium state if $x_{0} \neq 0$. Various authors considered the decay in this system starting only from one point $x_{0}=0$. In this case, the deterministic decay time is infinity and the action of the noise decreases the decay time in accordance with known scaling laws [12-16]. On the other hand, the effect of the NDD always appears when we consider any $x_{0} \neq 0$. In this case the decay times (both MFPT and NLRT) growth with noise, reach some maximum, and decrease. This effect was predicted theoretically in references [7, 8]. Before we consider the analytical expressions for MFPT and NLRT 
let us describe in more detail their definitions. These definitions involve the decision interval restricted by one or two boundaries which specify the area of the unstable state. The MFPT is the average time that the Brownian particle stays within the decision interval before it crosses any of the absorbing boundaries for the first time. Due to the absorbing boundary conditions, the MFPT neglects that the particle may return into the decision interval after it has crossed the boundary once. On the other hand, the NLRT is an average time which takes into account this inverse flow directed inside the decision interval, when the particle comes back. The definition of MFPT is well known and it is a very useful time characteristic of decay processes, because the analytical expression for MFPT can be written in quadratures for an arbitrary potential profile (See e.g. references [19]-[23]). Let $P(t)$ be the probability that the particle is within the decision interval. Initially $P(0)=1$. With time the particle escapes from the unstable state, therefore $P(\infty)=0$. If during the decay process the particle may cross the boundaries of the decision interval any number of times, the definition of the NLRT reads

$$
\tau=\int_{0}^{\infty} P(t) d t
$$

If the particle can cross the boundaries of the decision interval only once, the time (3) coincides with the MFPT. Otherwise the expression (3) takes into account the inverse probability current across the boundaries of the decision interval and differs from the MFPT. Therefore the MFPT is a particular case of the NLRT (3) , namely, the case in which we neglect the inverse probability current. The analytical expressions for NLRT in quadratures for an arbitrary potential were obtained in [21].

All numerical simulations shown in the paper were done using the algorithms described in [24], using the Heun algorithm for the stochastic integration, and the Ziggurath and a carry and subtract algorithm for the generation of the noise deviates. The integration time step used and the number of averages taken depend on the actual numerical experiment carried out, and on the set of parameters studied. Both have been changed throughout the numerical experiments aiming at optimizing the simulations: for example, when the noise intensity $q$ was changed, the integration time step $h$ was varied accordingly, to keep the quantity $q h$ small, which is a prerequisite to have good convergence in the Heun integrator.

\section{Decay times for the symmetric potential}

For the symmetric potential profile $\Phi(-x)=\Phi(x)$, the MFPT equals to (See e. g. [23, 25])

$$
T\left(x_{0}, L ; q\right)=\frac{\eta}{q} \int_{x_{0}}^{L} e^{\Phi(v) / q} \int_{0}^{v} e^{-\Phi(u) / q} d u d v .
$$

Let us consider the polynomial potential profile

$$
\Phi(x)=-a x^{k} / k,
$$




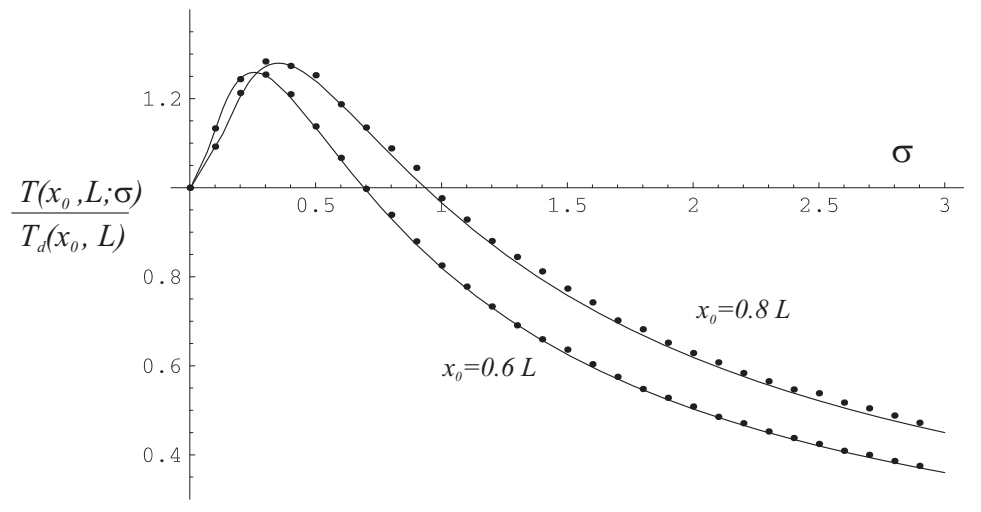

Figure 1. The MFPT normalized to the deterministic decay time versus the dimensionless temperature $\sigma=q /|\Phi(L)|$ for non-equilibrium states described by parabolic potential, for two initial states: $x_{0}=0.6 \mathrm{~L}$ and $x_{0}=0.8 \mathrm{~L}$. Symbols are the results of numerical simulations and solid lines are the theoretical results obtained from equation (8).

where $k=2 n$ is even. The exact expression for MFPT (44) with potential (5) was obtained in [12]) for a particular case, when the initial state is unstable equilibrium one $x_{0}=0$

$$
T(0, L ; q)=\frac{\eta L^{2}}{2 q}{ }_{2} F_{2}\left(1, \frac{2}{k} ; 1+\frac{1}{k}, 1+\frac{2}{k} ; \frac{\Phi(L)}{q}\right),
$$

where ${ }_{2} F_{2}\left(a_{1}, a_{2} ; b_{1}, b_{2} ; z\right)$ is a generalized hypergeometric function [26, 27]. It follows from (44), that for an arbitrary $x_{0}\left(-L<x_{0}<L\right)$ the MFPT reads

$$
T\left(x_{0}, L ; q\right)=T(0, L ; q)-T\left(0, x_{0} ; q\right),
$$

For example, if the potential is parabolic, then $k=2$ and MFPT is

$$
\begin{aligned}
& T\left(x_{0}, L ; q\right)=\frac{\eta}{2 q}\left[L^{2}{ }_{2} F_{2}\left(1,1 ; \frac{3}{2}, 2 ; \frac{\Phi(L)}{q}\right)-\right. \\
& \left.x_{02}^{2} F_{2}\left(1,1 ; \frac{3}{2}, 2 ; \frac{\Phi\left(x_{0}\right)}{q}\right)\right] .
\end{aligned}
$$

When the noise is absent the MFPT coincides with the deterministic decay time $T_{d}$. In particular, for the parabolic potential the deterministic decay time is

$$
T_{d}\left(x_{0}, L\right)=(\eta / a) \ln \left(L / x_{0}\right) .
$$

The plots of the MFPTs normalized to $T_{d}$ for the parabolic potential and for different $x_{0}$ are shown in figure 1 , where the symbols are the results of digital simulations and solid lines are the theoretical predictions. One can see that the NDD effect appears. The agreement between theory and numerical simulation is very good. To take into account the influence of the inverse probability current on the decay time we should consider NLRT. To obtain the NLRT we use the exact expression in quadratures obtained in references [8, 21] for the symmetric potential

$$
\tau\left(x_{0}, L ; q\right)=T\left(x_{0}, L ; q\right)+\Theta(L ; q),
$$




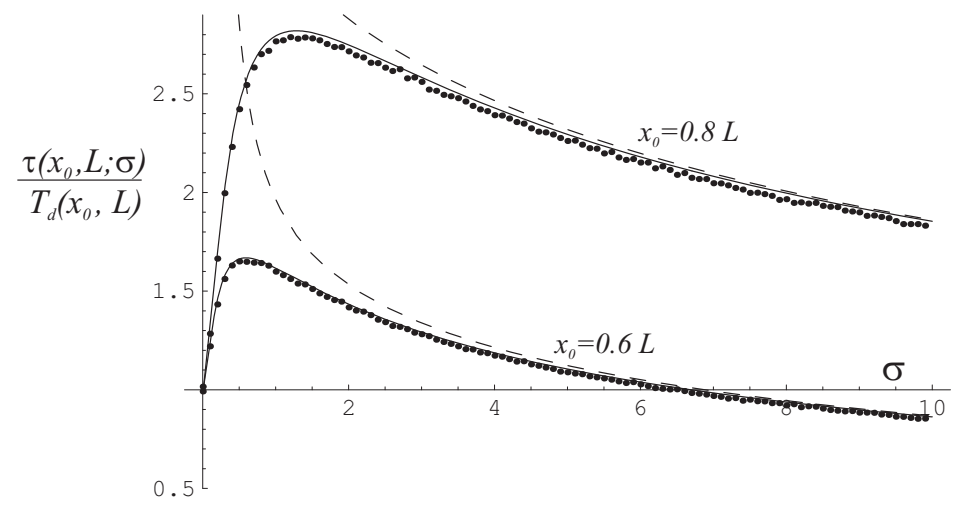

Figure 2. The normalized NLRT versus the dimensionless temperature $\sigma=q /|\Phi(L)|$ for non-equilibrium states described by a parabolic potential, for two initial conditions, namely $x_{0}=0.6 L$ and $x_{0}=0.8 L$. Symbols are the results of numerical simulations and solid lines are the theoretical predictions.

where $T\left(x_{0}, q\right)$ is the MFPT (4) and

$$
\Theta(L ; q)=\frac{\eta}{q} \int_{L}^{\infty} e^{\Phi(v) / q} d v \int_{0}^{L} e^{-\Phi(v) / q} d v .
$$

For the polynomial potential (5) the NLRT correction (10) reads

$$
\Theta(L ; q)=\frac{\eta L^{2}}{q}(-1)^{-1 / k}(-\sigma)^{\frac{2}{k}} \Gamma\left(\frac{1}{k} ; \sigma^{-1}\right) \gamma\left(\frac{1}{k} ;-\sigma^{-1}\right),
$$

where $\gamma(\alpha ; a)$ is the incomplete gamma function [27] and $\sigma=q /|\Phi(L)|$. In particular, for the parabolic potential profile (2), when $k=2$, the NLRT correction can be expressed in terms of error functions as

$$
\Theta(L ; q)=\frac{\eta \pi}{2 a}\left(1-\operatorname{Erf}\left(\sigma^{-1 / 2}\right)\right) \operatorname{Erfi}\left(\sigma^{-1 / 2}\right)
$$

The plots of NLRT are shown in figure 2, where symbols are the results of digital simulations and solid lines are the theoretical predictions. The NLRT increases with noise and displays the NDD effect. The enhancement of NLRT by noise is much greater than that of MFPT. This is because of the influence of the inverse probability current. Indeed, the NLRT correction (10) is positive for any $q>0$.

Note that the NLRT may exceed its deterministic value for a great range of the noise temperature, e. $\mathrm{g}$. for $x_{0}=0.6 L$ this range is $0<q<6|\Phi(L)|$ and for $x_{0}=0.8 L$ this range is more than one decade of dimensionless temperature. The value $|\Phi(L)|$ is the maximal height of the potential profile within the decision interval. Thus, the NDD effect takes place for the NLRT even for a strong noise intensity, while for the MFPT it appears only for weak noise when $q<|\Phi(L)|$. When $q>3|\Phi(L)|$ the asymptotical expression may be obtained for NLRT

$$
\begin{aligned}
& \tau\left(x_{0}, L ; q\right) \approx \frac{\eta}{a L^{k-2}}\left[\Gamma\left(\frac{1}{k}\right) \sigma^{-1+1 / k}-\right. \\
& \left.\frac{k}{2}\left(1+m^{2}\right) \sigma^{-1}+\frac{\Gamma\left(\frac{1}{k}\right)}{k+1} \sigma^{-2+1 / k}+0\left(\sigma^{-2}\right)\right],
\end{aligned}
$$




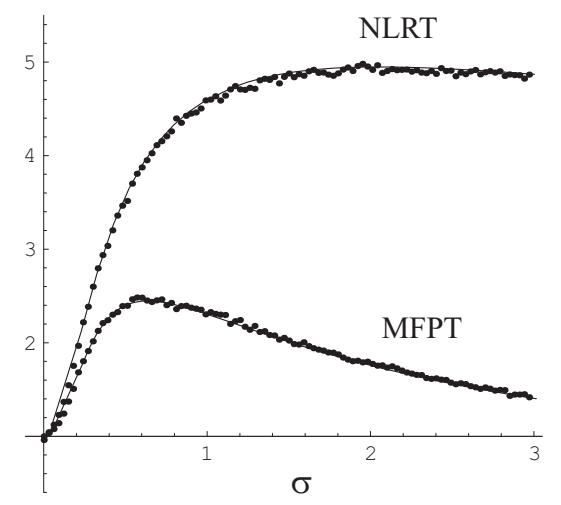

Figure 3. The normalized MFPT and NLRT versus temperature $\sigma=q /|\Phi(L)|$ for non-equilibrium state described by the cubic potential $\Phi(x)=-x^{3} / 3$, afor $x_{0}=0.8 L$. Symbols are the results of numerical simulations and solid lines are the theoretical predictions.

where $m=x_{0} / L$. The asymptotic (13), normalized to the deterministic decay time $T_{d}\left(x_{0}, L\right)$ is shown in figure 2 by dashed curve. It follows from equation (13) that when $\sigma \gg 1$, the NLRT does not depend on the initial conditions, i.e. on $x_{0}$. This is not the case for MFPT, which is

$$
T\left(x_{0}, L ; q\right) \approx \frac{\eta k}{2 a L^{k-2}}\left(1-m^{2}\right) \sigma^{-1},
$$

for $\sigma \gg 1$. We can not see this effect from figure 2 because the NLRT is normalized to different deterministic decay time. Therefore we may conclude that for strong noise intensity, the inverse probability current removes differences in decay times caused by initial conditions.

\section{Asymmetric potential}

Let us refer now to the asymmetric potential profile and consider the polynomial potential (5) where $k=2 n+1$ is odd. All the states $x_{0}>0$ of this system are nonequilibrium. In reference [12] the exact and approximate expressions for MFPT were investigated for $x_{0} \leq 0$ but not for $x_{0}>0$. While when $x_{0}>0$ the effect of NDD appears. We consider the case $x_{0}>0$. Let the decision interval be $R:[-\infty ; L]$, then the MFPT reads

$$
T\left(x_{0}, L ; q\right)=\frac{\eta}{q} \int_{x_{0}}^{L} e^{\Phi(v) / q} \int_{-\infty}^{v} e^{-\Phi(u) / q} d u d v
$$

and the NLRT, in accordance with [21], is given by equation (9) where

$$
\Theta(L ; q)=\frac{\eta}{q} \int_{L}^{\infty} e^{\Phi(v) / q} d v \cdot \int_{-\infty}^{L} e^{-\Phi(u) / q} d u .
$$

Using the expression for $T(0, L ; q)$ obtained in [12] it is possible to write the analytic expression for MFPT when $x_{0}>0$

$$
T\left(x_{0}, L ; q\right)=\frac{\eta}{2 q}\left[L_{2}^{2} F_{2}\left(1, \frac{2}{k} ; 1+\frac{1}{k}, 1+\frac{2}{k} ; \frac{\Phi(L)}{q}\right)\right.
$$




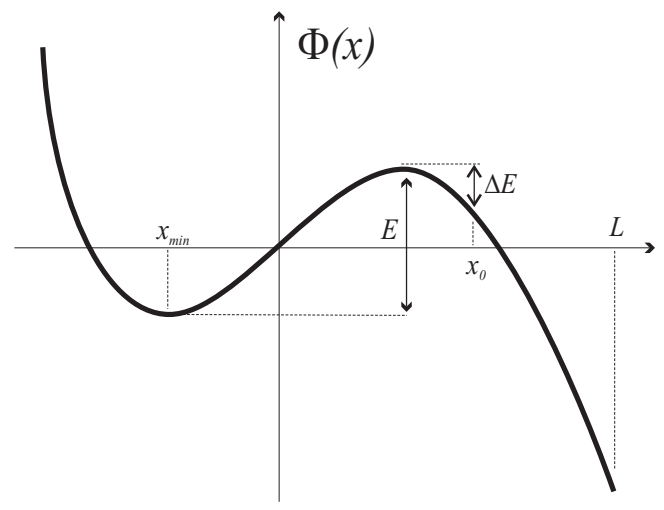

Figure 4. Potential profile (19).

$$
\begin{aligned}
& -x_{02}^{2} F_{2}\left(1, \frac{2}{k} ; 1+\frac{1}{k}, 1+\frac{2}{k} ; \frac{\Phi\left(x_{0}\right)}{q}\right) \\
& \left.+\frac{k^{-2+2 / k}}{q}\left(\frac{q}{a}\right)^{\frac{2}{k}} \Gamma\left(\frac{1}{k}\right) \gamma\left(\frac{1}{k} ; \frac{-\Phi(L)}{q}, \frac{-\Phi\left(x_{0}\right)}{q}\right)\right] .
\end{aligned}
$$

The NLRT correction (16) for the polynomial potential (5) is

$$
\Theta(L ; q)=(-1)^{1+1 / k}\left(\frac{\eta}{q}\right)\left(\frac{1}{k}\right)^{2}\left(\frac{q k}{a}\right)^{2 / k} \Gamma\left(\frac{1}{k} ;-\sigma^{-1}\right) \Gamma\left(\frac{1}{k} ; \sigma^{-1}\right)
$$

The plots of MFPT (17) and NLRT (9) for a cubic potential $(k=3)$ and $x_{0}=0.8 \mathrm{~L}$ are presented in figure 3 , where the symbols are the results of numerical simulation. We see that the NDD takes place. The NLRT is always greater than the MFPT because the inverse probability current always increase the decay time. In general, despite the apparent difference between the expressions for decay time in antisymmetric potentials, the features of the times are similar.

\section{Potential with barrier}

Let us consider now a smooth potential profile with a barrier

$$
\Phi(x)=-\frac{a x^{3}}{3}+b x
$$

where $a=1, b=1$ and boundary is located at the point $L=5$ (See the figure 4). Then, the local minimum of this profile is located at $x=-1$, and the local maximum at $x=1$. The barrier height is $E=\Phi(1)-\Phi(-1)=4 / 3$. The expressions in quadratures for MFPT and NLRT for potential profile of this type were obtained in [8, 21]. They coincide with the corresponding expressions for the antisymmetrical potential (91), (15), and (16). We will consider two cases of initial positions: $x_{0}=1.5$ and $x_{0}=3$. Both of these initial states are situated behind the potential barrier. The barrier height "seen" by the Brownian particle in its initial position $x_{0}$ is $\Delta E=\Phi(1)-\Phi\left(x_{0}\right)$ (see figure 4). Therefore $\Delta E / E<1$ for $x_{0}=1.5$ and $\Delta E / E>1$ for $x_{0}=3$. In references [4, [5] it was shown that the behaviours of MFPT and NLRT have strong dependence on the value 


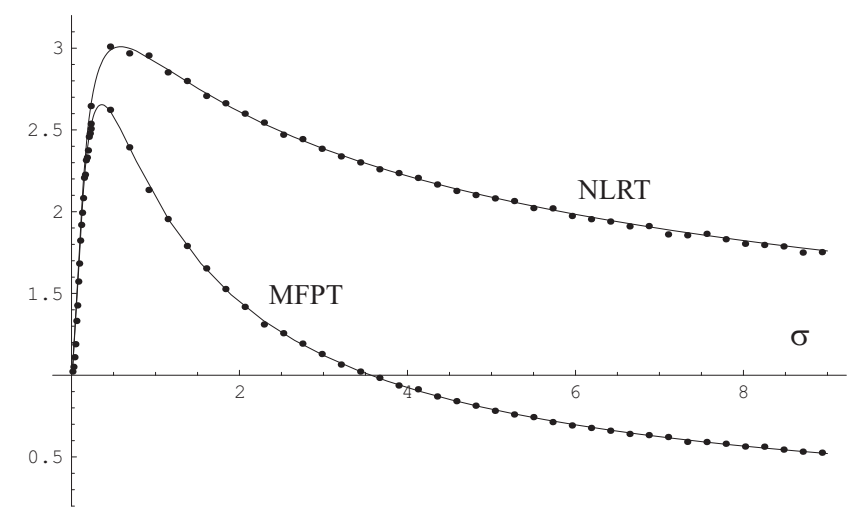

Figure 5. The normalized MFPT and NLRT versus the dimensionless temperature for potential profile with the barrier (19) and for non-equilibrium initial unstable state $x_{0}=3(\Delta E>E)$. Symbols are the results of numerical simulations and solid lines are the theoretical predictions.

$\Delta E / E$. Indeed, if $\Delta E / E>1$, in the deterministic limit (i. e. when $q \rightarrow 0$ ) we obtain a finite value for the escape time

$$
T_{d}\left(x_{0}, L\right)=\eta \ln \left[\left(\frac{x_{0}+1}{L+1}\right)\left(\frac{L-1}{x_{0}-1}\right)\right]^{1 / 2} .
$$

By increasing the noise intensity the particles can go towards the well and be delayed there. As a consequence, the MFPT and the NLRT increase with $q$ and go through a maximum for some $q^{*}>0$. This is the typical case of NDD phenomenon considered above (see figure 5). However, if $\Delta E / E<1$ (see figure 6) the situation becomes more complicated. It is evident that if there is no noise $(\xi=0)$, the escape times (both MFPT and NLRT) are equal to the deterministic escape time (201). At the same time, it follows from equation (15) that in the limit of $q \rightarrow 0$, the MFPT and the NLRT go to infinity and for $q=0$ a singularity appears. This singularity was demonstrated for the first time in references [4, 5. The asymptotic expression for the MFPT as $q \rightarrow 0$ and $E>\Delta E>0$ can be obtained from equation (15):

$$
T\left(x_{0} ; q\right) \approx \sqrt{\frac{2 \pi q}{\Phi^{\prime \prime}\left(x_{\min }\right)}} \frac{\eta}{\left(-\Phi^{\prime}\left(x_{0}\right)\right)} \exp \left(\frac{E-\Delta E}{q}\right),
$$

where $x_{\min }$ is the coordinate of the local minimum. For both above cases the plots of MFPT and NLRT are shown in figures 5 and 6 , where the symbols are the results of digital simulations and solid lines are the theoretical predictions. One can see, that the agreement between theory and the results of numerical simulations is good only for initial position $x_{0}=3$ and there is strong difference when $x_{0}=1.5$. In the last case, when $q \rightarrow 0$, the theoretical curve goes to infinity, while digital simulation gives the deterministic time. We can interpret this effect as follows: as explained in reference [5] the theoretical curve goes to infinity because even a very small noise intensity can eventually push the particle, initially located behind the barrier, back into the potential well. Then, the particle will be trapped there for a long time, because the well is very 


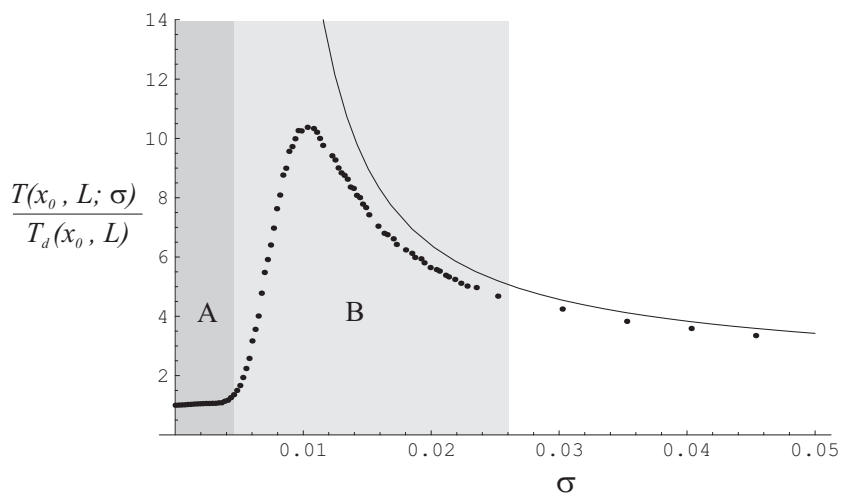

Figure 6. The normalized MFPT versus the dimensionless temperature $\sigma=q / E$ for potential profile with the barrier (19) and for non-equilibrium initial unstable state $x_{0}=1.5(\Delta E<E)$. Symbols are the results of numerical simulations and solid lines are the theoretical predictions.

deep. These trajectories of the Brownian particles lead to the singularity of the MFPT for $q \rightarrow 0$. On the other hand, the observation time in a digital simulation is finite and for some value of $q$ it becomes smaller than the average escape time of a particle trapped in the well. Therefore the simulated escape time has the maximum at this point (See the region $\mathrm{B}$ in figure 6). Besides, the ensemble of particles in a numerical experiment is also finite. That is why for very small $q$, when the probability for a particle to be trapped in the well decreases exponentially to zero, we do not observe such particles in the simulation. As a consequence, the average decay time in simulation becomes equal to the deterministic one at $q \rightarrow 0$ (See the region $\mathrm{A}$ in the figure 6 ).

\section{Conclusions}

We have derived the exact expressions for MFPT and NLRT in the case of polynomial potential profiles and for arbitrary unstable non-equilibrium initial positions. Expressions for MFPT obtained earlier (See e.g. [12]) are valid only for the particular case when the initial position is the unstable equilibrium state and the action of noise always decreases the decay times. We have obtained nonmonotonic behaviour of the decay times as a function of the noise intensity and have shown that the inverse probability current can increase the NLRT with respect to the MFPT. Moreover the range in which we obtain the NDD effect is larger for the NRLT than for the MFPT. An important characteristic of the NDD effect is that we can both accelerate or slow down the decay of unstable nonequilibrium states by varying the intensity of fluctuations in a larger range for NRLT than for MFPT. The numerical simulations are in good agreement with the theory for most of potentials and initial conditions considered, except the specific case of singularity in the MFPT and the NLRT for $q \rightarrow 0$. This singularity appears for the non-equilibrium states in a potential profile with a barrier when $\Delta E<E$ (figure 6) and it is also responsible for the enhancement of the stability of 
fluctuating metastable states [5, 9, 10]. In this case due to the limitation in the particles number and observation time in simulation, we cannot obtain the singularity (infinite decay time) numerically. Therefore one should expect that the same difference found between theoretical values of the average decay times and numerical simulations for $\Delta E<E$ and $q \rightarrow 0$ will appear in experimental results when compared with theoretical ones.

\section{Acknowledgments}

This work has been supported in part by INTAS Grant 01-450, by INFM and MIUR, and by RFBR (Project Nos. 02-02-17517, 1729.2003.2).

\section{References}

[1] Gammaitoni L, P. Hänggi P, Jung P, Marchesoni F, Rev. Mod. Phys. 70223 (1998); Mantegna R N, Spagnolo B, Trapanese M, Phys. Rev. E 63011101 (2001); Dykman M I, Luchinsky D G, Mannella R, McClintock P V E, Stein N D, and Stocks N G, Nuovo Cimento D 17661 (1995)

[2] Doering C R, Gadoua J C, Phys. Rev. Lett. 692318 (1992); Mantegna R N, Spagnolo B Phys. Rev. Lett. 843025 (2000); Mantegna R N, Spagnolo B J. Phys. IV 8247 (1998); Dykman M I, Rabitz H, Smelyanskiy V N, and Vugmeister B E, Phys. Rev. Lett. 791178 (1997); Soskin S M, Sheka V I, Linnik T L, and Mannella R Phys. Rev. Lett. 861665 (2001); Soskin S M, Mannella R, Arrayas M, and Silchenko A N, Phys. Rev. E, 63051111 (2001); Smelyanskiy V N, Golding B, Luchinsky D G, Mannella R, McClintock P V E, and Dykman M I, Chaos 11587 (2001)

[3] Mantegna R N, Spagnolo B, Phys. Rev. Lett. 76563 (1996); Int. J. Bifurcation and Chaos 8 783 (1998); in Freund I A, Poeschel T (Eds.), Stochastic Processes in Physics, Chemistry and Biology, Lecture Notes in Physics 2000, (Springer, Berlin) p 327; Stocks N G and Mannella R, Phys. Rev. Lett. 804835 (1998)

[4] Agudov N V and Spagnolo B, AIP Conference Proseedings, 502 272-277, eds. Broomhead D S, Luchinskaya E A, McClintock P V E, Mullin T 1999 (Melville, New York)

[5] Agudov N V and Spagnolo B, Phys. Rev. E 64 035102(R) (2001)

[6] Agudov N V and Malakhov A N, Int. J. Bifurcation and Chaos 5531 (1995)

[7] Agudov N V, Phys. Rev. E 572618 (1998)

[8] Agudov N V and Malakhov A N, Phys. Rev. E 606333 (1999)

[9] Agudov N V, Dubkov A A, Spagnolo B, Physica A 325144 (2003)

[10] Fiasconaro A, Valenti D, Spagnolo B, Physica A 325136 (2003)

[11] Wackerbauer R, Phys. Rev. E 592872 (1999)

[12] Arecchi F T, Politi A, and Ulivi L, Nuovo Cimento B 71119 (1982)

[13] Haake F, Haus J W, and Glauber R, Phys. Rev. A 233255 (1981)

[14] Colet P, San Miguel M, Casademunt J, and Sancho J M, Phys. Rev. A 39149 (1989); F. de Pasquale, J. M. Sancho, M. San Miguel and P. Tartaglia, Phys. Rev. Lett. 562473 (1986); J. I. Jimenez Aquino, J. M. Sancho and J. Casademunt, Physica A 195163 (1993); J. I. Jimenez Aquino and J. M. Sancho, Phys. Rev. E 4716558 (1993).

[15] Colet P, de Pasquale F, and San Miguel M, Phys. Rev. A 435296 (1991)

[16] Suzuki M, Prog. Theor. Phys. 56, 77 (1976); Phys. Lett. A 67339 (1978)

[17] de Pasquale F and Tombesi P, Phys. Lett. A 727 (1979)

[18] Ciuchi S, de Pasquale F and Spagnolo B, Phys. Rev. E 473915 (1993)

[19] Stratonovich R L 1963 Topics in the Theory of Random Noise Vol. 1 (Gordon and Breach, New York)

[20] Hänggi P, Talkner P, and Borcovec M, Rev. Mod. Phys. 62251 (1990) 
[21] Malakhov A N, CHAOS 7488 (1997)

[22] Malakhov A N and Pankratov A L, Adv. in Chem. Phys. 121 357-438 (2002)

[23] Pontryagin L S, Andronov A A, and Vitt A A, Zh. Eksp. Teor. Fiz. 3165 (1933) [in Moss F and McClintock P V E (Eds.), Noise in Nonlinear Dynamics vol 1 (Cambridge University Press, Cambridge, 1989) p 329]

[24] Mannella R, Int J. Mod. Phys. C 13 1177-1194 (2002).

[25] Gardiner C W 1985 Handbook of Stochastic Methods, (2nd Edition, Springer, Berlin)

[26] Grandshteyn I S and Ryzhik I M 1980 Table of Integrals, Series, and Products. (Academic Press, New York)

[27] Abramowitz M and Stegun I A 1972 Handbook of Mathematical Functions. (Dover Publications, Inc., New York) 